\title{
Editorial
}

Gynäkologe 2021 · 54:242-243

https://doi.org/10.1007/s00129-021-04786-5

Angenommen: 2. März 2021

๑) Springer Medizin Verlag GmbH, ein Teil von Springer Nature 2021

Klaus Diedrich ${ }^{1}$ Stefanie Schumacher-Schmidt ${ }^{2}$

${ }^{1}$ Klinik für Frauenheilkunde, Universitätsklinikum Schleswig-Holstein, Lübeck, Deutschland

${ }^{2}$ Journals and ePublishing, Springer Medizin Verlag GmbH, Heidelberg, Deutschland

\section{Facharzt-Training Frauenheilkunde und Geburtshilfe - Das zweite Sonderheft ist da!}

\section{Weitere praxisnahe Fallbeispiele zur Prüfungsvorbereitung}

\section{Liebe Leser*innen,}

Im November 2020 haben wir Ihnen das erste Sonderheft „Facharzt-Training Frauenheilkunde und Geburtshilfe" vorgestellt. Herr Professor Diedrich (federführend), Herr Professor Friedrich, Herr Professor Gembruch, Herr Professor Jonat und Herr Professor Rody, die Heftherausgeber, hatten für die Vorbereitung auf die Facharztprüfung, gemeinsam mit ausgewählten Expert*innen, mit 25 Fällen den Beginn einer Fortbildungsreihe geschaffen. Diese vereint umfassend, aber übersichtlich kompakt und dabei in ganz neuartiger Form klinische Alltagsexpertise mit essenziellen Wissensgrundlagen anhand von konkreten Fallbeispielen.

Nun freuen wir uns, Ihnen weitere 21 Fallvignetten im gewohnten Konzept vorzustellen (• Abb. 1). Die Autor*innen präsentieren Ihnen die vielfältigen Neue-

\begin{tabular}{|l}
\hline Dankeschön! \\
\hline Unser herzlicher Dank gilt allen Autor*innen, \\
die sich an diesem Projekt so engagiert \\
beteiligt und aus ihrem Spezialgebiet \\
praxisnahe Patientinnenbeispiele \\
aufgearbeitet haben. \\
Wir danken außerdem herzlich den \\
Heftherausgebern für die Erarbeitung \\
dieser außergewöhnlichen, spannenden, \\
lehrreichen und dabei unterhaltsamen \\
Fortbildungsreihe.
\end{tabular}

rungen in unserem Fach in einer ansprechenden und lernbaren Form. Typische und alltagsnahe Fallbeispiele wurden systematisch und aktuell aufbereitet. Der Start in jedes Kapitel ist dabei wieder eine klinische Fragestellung, an die sich dann der gesamte klinische Wissensinhalt anschließt.

Das „Facharzt-Training Frauenheilkunde und Geburtshilfe" umfasst somit nun 46 praxisorientierte Fallbeispiele und deckt das relevante Wissen für die Facharztprüfung Frauenheilkunde und Geburtshilfe prüfungsgerecht und praxisnah ab. Sicher können auch langjährig erfahrene Gynäkolog*innen und Geburtshelfer*innen von dieser Darstellung profitieren und neue Einblicke gewinnen.

\section{Aufbau nach Spezialitäten}

Die Frauenheilkunde und Geburtshilfe ist ein vielfältiges und umfangreiches Fach, das aus 5 verschiedenen Spezialitäten besteht: die allgemeine Gynäkologie, die Gynäkologische Onkologie, die Gynäkologische Endokrinologie und Reproduktionsmedizin, die Urogynäkologie sowie die Geburtshilfe, Prä- und Perinatalmedizin. Dementsprechend ist das Facharzt-Training aufgebaut. 


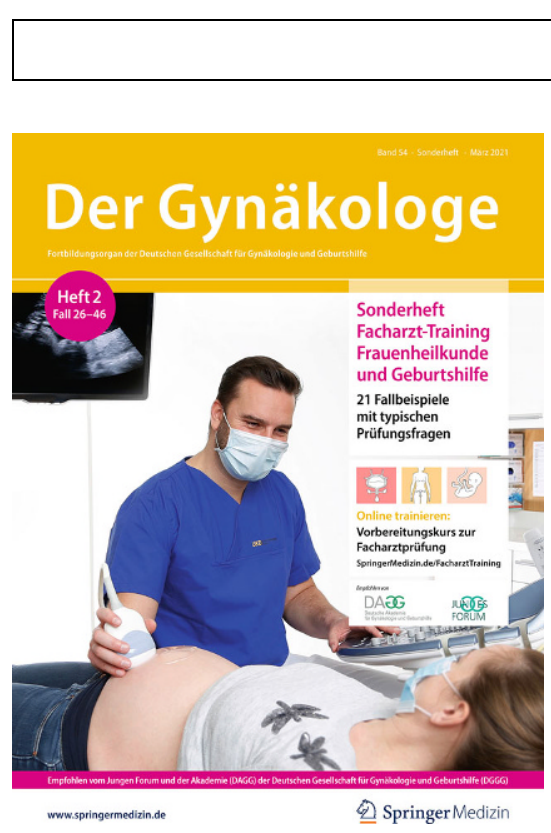

Abb. $1 \Delta$ Das 2. Sonderheft „Facharzt-Training Frauenheilkunde und Geburtshilfe" ist da!

\section{Empfehlung der Gesellschaft}

Es freut uns ganz besonders, dass das Junge Forum (JF) und die Akademie (DAGG) der Deutschen Gesellschaft für Gynäkologie und Geburtshilfe (DGGG) dieses Facharzt-Training zur Prüfungsvorbereitung empfehlen.

\section{Sonderaktion}

Im Aktionszeitraum erhalten Sie noch bis zum 30. April 2021 je ein Sonderheft für $22 €$ (statt $44 €$ ) oder beide Hefte im Paketpreis für nur $44 €$ (statt $75 €$ ).

Bitten wenden Sie sich dazu an MarieLuise Witschel (Marie-Luise.Witschel@ springer.com) unter Angabe des folgenden Aktionscodes: C0019579.

\section{Alle Inhalte als E-Learning verfügbar}

Zusätzlich zu den beiden Sonderheften stehen alle 46 Fallvignetten passend $\mathrm{zu}$ den Heftbeiträgen auch als E-Learning-Kurse auf www.springermedizin.de/ facharzttraining unter ,Facharzt-Training Frauenheilkunde und Geburtshilfe" zur Verfügung. So haben Sie alles Wichtige zur Prüfungsvorbereitung überall und jederzeit dabei.
Wir wünschen Ihnen viel Freude bei der Lektüre dieser regulären Ausgabe von Der Gynäkologe und - wenn Sie mögen der beiden Sonderhefte.

Ihre

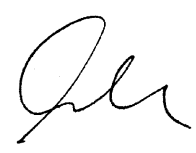

\section{Klaus Diedrich}

S6. Solvernaller-Schmicht

\section{Stefanie Schumacher-Schmidt}

\section{Korrespondenzadresse}

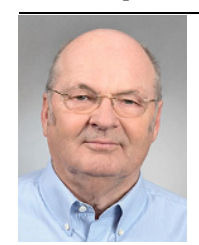

Prof. Dr. med. Dr. h.c. mult. Klaus Diedrich

Klinik für Frauenheilkunde, Universitätsklinikum Schleswig-Holstein Ratzeburger Allee 160, 23538 Lübeck, Deutschland Prof.Diedrich@gmail.com

Interessenkonflikt. K. Diedrich und S. SchumacherSchmidt geben an, dass kein Interessenkonflikt besteht.
GBA: 1088 Anträge zur Erbringung einer Zweitmeinung gestellt

Die KBV hat die Zweitmeinungsanträge bis Juni 2020 ausgewertet: Besonders häufig wurde eine Genehmigung als zweitmeinender Arzt für Hysterektomien nachgefragt. Der Großteil der Anträge ist bewilligt worden.

Berlin. Seit Inkrafttreten der Zweitmeinungsrichtlinie für drei Indikationen im Dezember 2018 wurde bis Juni 20201088 mal ein Antrag auf Erbringung dieser Leistung von Ärzten gestellt. Mehr als die Hälfte aller Anträge - 566 - betrafen dabei Hysterektomien. 284 mal beantragten Ärzte, als Zweitmeiner für Tonsillektomien tätig werden zu dürfen, 238 mal für Schulterathroskopien.Seit 2020 können GKVVersicherte auch eine Zweitmeinung vor einer Implantation von Knieendoprothesen einholen. Diese Indikation ist aber in dem jetzt erstmals von der KBV erstellten und vom Gemeinsamen Bundesausschuss (GBA) veröffentlichten Bericht noch nicht enthalten.

Der weitaus größte Teil der Anträge wurde positiv beschieden. Abgelehnt wurden 21 Anträge bei Tonsillektomien, 64 bei Hysterektomien und 10 bei Schulterathroskopien. Häufigster Grund für eine Ablehnung war die fehlende Weiterbildungs- oder Lehrbefugnis des Arztes. „Interessenkonflikte oder keine ausreichende Unabhängigkeit der Antragsteller tauchten in der Untersuchung hingegen kein einziges Mal als Grund für eine Ablehnung auf", heißt es beim GBA. Anträge auf Genehmigung der Leistungserbringung wurden zum überwiegenden Teil von Vertragsärzten gestellt. Nur 61 Anträge kamen von Krankenhausärzten und zwei von Privatärzten. Ärzte, die eine Zweitmeinung abrechnen wollen, müssen die in der entsprechenden Richtlinie festgelegten besonderen eingriffsspezifischen Qualifikationen nachweisen. Die Genehmigungen, diese Leistungen abzurechnen, erteilen die Kassenärztlichen Vereinigungen. (chb)

Quelle: www.aerztezeitung.de, 22.02.2021 